\title{
A Novel Technique for Removal of High Density White Spot Noise from Digital Neutron Radiographic Images
}

\author{
A. Hindasyah ${ }^{1,2^{\star}}$, D. Sudiana ${ }^{1}$ and D. Gunawan ${ }^{1}$ \\ ${ }^{I}$ University of Indonesia, Jl. Margonda Raya, Depok 16424, Indonesia \\ ${ }^{2}$ Center for Science and Technology of Advanced Materials, National Nuclear Energy Agency, \\ Puspitek Area, Serpong, Tangerang Selatan 15310, Indonesia
}

\section{ARTICLE INFO}

\section{Article history:}

Received 27 March 2018

Received in revised form 29 June 2018

Accepted 29 June 2018

Keywords:

Neutron radiography

Neutron tomography

White spot noise

Switching filter

Neutron imaging

\begin{abstract}
A B S T R A C T
This paper proposes a novel technique of adaptive switching alternative median (ASAM) filter for high-density white spot noise removal. The ASAM filter is composed of two blocks filtering, namely main and secondary block filtering, respectively. The proposed secondary block filtering is a new technique in highdensity impulse noise removal and the main contribution of this research. The ASAM algorithm was tested on the standard 8-bit gray-scale, $512 \times 512$ pixel Lena image and a real neutron radiographic image. The results showed significant reduction of white spot noise in both types of images through visual inspection. To measure the performance of noise removal in simulation test we measured the peak signal-to-noise ratio (PSNR), structural similarity (SSIM) index, and denoising time, and in real application tests we measured signal-to-noise ratio (SNR). From the experiments of simulation test, at the highest level noise of $95 \%$ the obtained PSNR and SSIM are $23.584 \mathrm{~dB}$ and 0.696 respectively. These are higher than the results of other algorithms that are $16.697 \mathrm{~dB}$ and 0.475 , respectively, for DBA, $16.696 \mathrm{~dB}$ and 0.408 for NAFSM, and $18.860 \mathrm{~dB}$ and 0.568 for NASNLM. The denoising times for DBA, NAFSM, NASNLM, and ASAM were obtained as $6.469 \mathrm{~s}, 5.186 \mathrm{~s}, 36.735 \mathrm{~s}$, and $5.197 \mathrm{~s}$ respectively. From the experiments of real application test we obtained the SNR for DBA, NAFSM, NASNLM, and ASAM as $32.42 \mathrm{~dB}, 6.01 \mathrm{~dB}, 18.77 \mathrm{~dB}$, and $32.96 \mathrm{~dB}$, respectively. In general, these results show that ASAM filter is superior to the existing filtering methods. The ASAM filter improved the image restoration quality, especially in removing the highdensity white spot noise, and was able to yield good filtering result which exhibits better PSNR, SSIM, denoising time, and qualitative visual inspection.
\end{abstract}

(C) 2018 Atom Indonesia. All rights reserved

\section{INTRODUCTION}

Neutron radiography has been widely used in various fields of research and Non-Destructive Testing (NDT) [1-3]. At the GA Siwabessy Multipurpose Reactor (RSG-GAS)-BATAN, an Indonesian research nuclear reactor, Neutron Radiographic Facility (NRF) was installed in 1992. The NRF is equipped with a scintillator sensor, a reflector mirror, and a CCD camera for digital data acquisition. To obtain images from NRF, the neutron beam is converted to a visible light sing a scintillating screen sensor [4,5]; then, the image of

*Corresponding author.

E-mail address: ahindasyah@batan.go.id

DOI: https://doi.org/10.17146/aij.2018.846 the sample on the scintillating screen is transmitted and reflected by a tilted mirror to a CCD camera.

Experimentally, CCD cameras must be validated for detector efficiency against parameters on which it depends, such as heat disturbance, gamma sensitivity, and stability [6]. Although the CCD camera is not irradiated directly, a large number of radiation particles hit the CCD detector and produce white spot noise (WSN) in the image. The WSN is also produced by secondary scattered neutron and gamma radiation hitting the CCD detector in spite of sophisticated shielding [7-9]. The WSN is an impulse noise which has random intensity, but generally brighter than the average pixel intensity in the radiographic image. It causes degradation of image resolution and loss of image 
detail, and potentially results in failure of further evaluations, especially in $2 \mathrm{D}$ and $3 \mathrm{D}$ tomographic reconstruction. Therefore, removal the WSN and preservation of the image details are crucial preprocessing in neutron imaging.

Recently, several techniques to remove the impulse noise was proposed [10-25]; however, not so much development occurs on the technique to remove WSN, since not all impulse noise removal algorithms can be used to remove WSN. One of the most commonly used methods is the median filtering, which replaces a pixel's center value in sliding window with the median value of its neighbors. A hybrid method between adaptive threshold and mean harmonic filtering was proposed [26]. However, for high-density noise, these methods could not be performed perfectly since median-based and mean harmonic filters restore each pixel with an approximate value regardless whether it is a noise or noise-free pixel. To improve the filtering, an algorithm that combines the Laplacian of Gaussian (LoG), adaptive threshold, and median filters was proposed [27]. The algorithm could satisfactorily eliminate WSN and retain the image details. However, it could not eliminate WSN with variable sizes, shapes, and occurrence. To overcome this drawback, the iterative procedure uses glitches and bad pixel algorithms was implemented [28]. However, this algorithm cannot be used in highdensity noise as this procedure only detects either the first or the last element of an array as outliers in sliding windows that has a maximum size of $5 \times 5$.

In order to address the above drawbacks, a novel technique using hybrid method between global threshold and adaptive switching alternative median (ASAM) filter is proposed. The technique has three stages: (1) separating noise from the image using a threshold, (2) marking noisy and noise-free pixels, and (3) removing the noise by the ASAM algorithm. The ASAM algorithm is developed from the switching adaptive median filter (SM) [29] followed by adding a secondary block filtering (SBF). The addition of SBF block is a new technique in high-density impulse noise removal and the main contribution of this study. The ASAM filter could remove high-density noise and give better peak signal-to-noise ratio (PSNR) and structural similarity (SSIM) index than the existing algorithm.

\section{EXPERIMENTAL METHODS}

The purpose of this study is to eliminate highdensity WSN on neutron radiographic images while preserving the detailed image information at the same time. The three stages that are proposed will be explained in the following modules.

\section{Noise intensity detection module}

WSN is a random value impulse noise that has different probabilities and random intensities. The main purpose of this module is to separate the noise from the image by converting a random value impulse noise into a fixed one using global threshold expressed in eq. (1).

$$
T=T_{0}\left(I_{\max }-I_{\min }\right)
$$

where $I_{\max }$ and $I_{\min }$ denote maximum and minimum pixel intensity, respectively. $T_{0}$ denotes a specified threshold value, and $T$ denotes the threshold value. The optimal value of $T_{0}$ in this experiment is $30 \%$. As expressed in eq. (2), the pixels that have greater value than the threshold $T$ are considered as noise pixels and replaced by a maximum of 255 for 8-bit image. The values of other pixels are maintained to preserve the image details.

$$
X(i, j)=\left\{\begin{aligned}
X(i, j), & X(i, j) \leq T \\
\text { maximum, } & X(i, j)>T
\end{aligned}\right.
$$

where $X(i, j)$ denotes the pixel intensity at position $(i, j)$.

Figures 1(a) and 1(b) show the histogram of radiographic image of a valve before and after threshold process, respectively. After threshold processing, the intensities of the noise are collected at the end of the noisy image histogram. In this case, the number of noisy pixels is 152.927 pixels or about $14.58 \%$ for optimal $T_{0}$ value of $30 \%$.

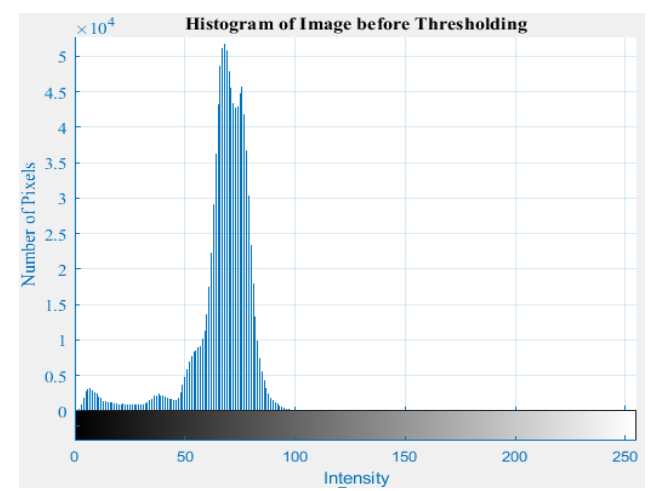

(a)

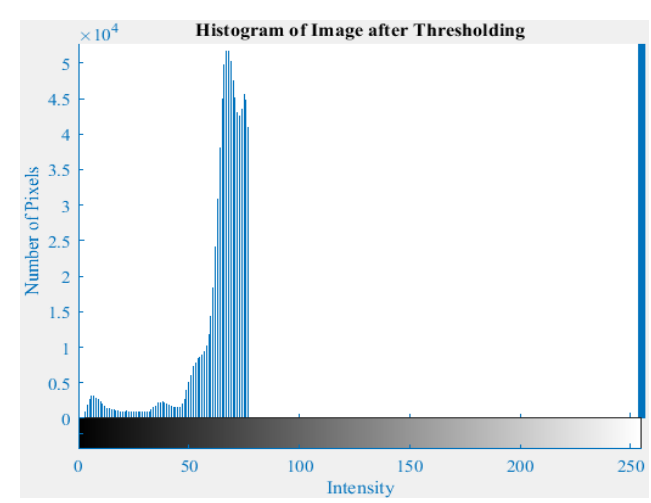

(b)

Fig. 1. Histogram of radiographic image (a) before thresholding (b) after thresholding with $T_{0}$ of $30 \%$. 


\section{Noise position detection module}

The module is used to locate noise and nonnoise pixels, respectively. Noise position detection is performed by thresholding the image using binary masking as expressed in eq. (3).

$$
B_{M}(i, j)=\left\{\begin{array}{lr}
0, & \text { if } X(i, j) \text { maximum } \\
1, & \text { if } X(i, j) \text { non }- \text { noise pixel }
\end{array}\right.
$$

where $B_{M}(i, j)$ denotes the binary mask value and $X(i, j)$ is the pixel intensity at position $(i, j)$. If $X(i, j)$ equals to the maximum value, then $X(i, j)$ is considered as noise, so that the value of $B_{M}(i, j)=0$. Otherwise, if the $X(i, j)$ not equal to maximum, then $X(i, j)$ is non-noise pixel, so that $B_{M}(i, j)=1$. Noise pixels are marked by black intensity, while non-noise pixels are marked by white intensity in the binary image, as shown in Fig. 2.

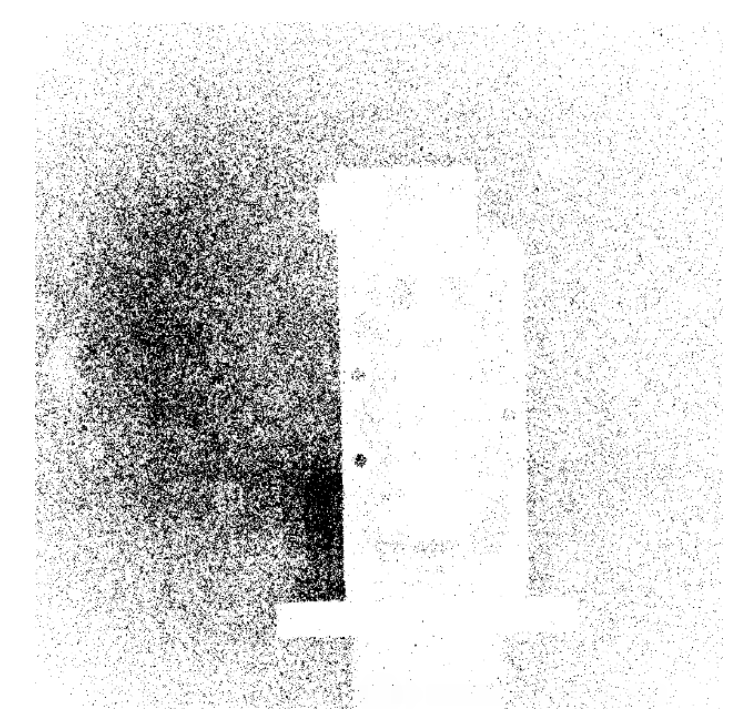

Fig. 2. Binary mask of image after thresholding with $T_{0}$ of $30 \%$.

\section{Noise cancellation module}

The noise cancellation module consists of two filtering blocks, namely main and secondary block filtering, respectively, as shown in Fig. 3. The mathematical model of the noise cancellation module is expressed in eq. (4),

$$
\begin{aligned}
& Y(i, j)=B_{M} \cdot X(i, j)+ \\
& \left(1-B_{M}\right) \cdot\left\{(1-a) \cdot M_{a}(i, j)+(1-b) \cdot M_{b}(i, j)\right\}
\end{aligned}
$$

where $Y(i, j)$ denotes the output pixel, $X(i, j)$ denotes the input pixel, $B_{M}$ denotes binary mask, $M_{a}(i, j)$ denotes a filtering process using main block filtering, $M_{b}(i, j)$ denotes a filtering process using secondary block filtering, and $a$ and $b$ denote alternative parameters for the main or secondary block, where $a=0$ and $b=1$ for the main block filtering or $a=1$ and $b=0$ for secondary block filtering. The filtering process uses a sliding window
$W_{2 f+1}$ with odd positive number of $(2 f+1) \times(2 f+1)$ dimensions given in eq. (5) [30].

$$
W_{2 f+1}(i, j)=X(i+m, j+n)
$$

where $m, n \in\{-f \ldots 0 \ldots+f)$.

If the centered pixel in the sliding window is a noise-free pixel $\left(B_{M}=1\right)$ then the output pixel is the same as the input pixel, $Y(i, j)=X(i, j)$. Otherwise, if it is a noise pixel $(B M=0)$, the output pixel becomes

$$
Y(i, j)=(1-a) \cdot M_{a}(i, j)+(1-b) \cdot M_{b}(i, j)
$$

Then, noise-free neighboring pixels $G_{2 f+l}(i, j)$ in the sliding window are calculated as:

$$
G_{2 f+1}(i, j)=\sum_{m, n=-f}^{f} N(i+m, j+n)
$$

where $N(i+m, j+n)=1$ for noise-free pixels. All of the "noise-free pixels" will be used as candidates for selecting the median pixel, given by eq. (8) [30].

$$
M(i, j)=\text { median }\{X(i+m, j+n)\}
$$

The first filtering process will by default utilize the main block filtering, and the size of sliding window is initialized to $3 \times 3$ or $f=1$. In main block filtering, if the current sliding window did not have a minimum number of three noise-free pixels (i.e., $G_{2 f+l}(i, j)<3$ ), then the sliding window will be expanded by one pixel (i.e. $f \leftarrow f+1$ ). This procedure is repeated until the criteria of $G_{2 f+l}(i, j) \geq 3$ and $f \leq 3$ are fulfilled. If the criteria in the main block have been fulfilled, the pixel at $(i, j)$ is replaced by median value, then the sliding window will be shifted to the next pixel, $f$ returns to the initial value and the ouput pixel becomes

$$
Y(i, j)=(1-a) \cdot M_{a}(i, j)
$$

Otherwise, if the criteria of the main block filtering are not fulfilled, then the filtering process is switched to the secondary block filtering. In secondary block filtering, the size of sliding window of $9 \times 9$ or $f=4$ is implemented. If the current sliding window does not have the minimum number of one noise-free pixel (i.e., $G_{2 f+l}(i, j)<1$ ), then the sliding window will be expanded by one pixel (i.e., $f \leftarrow \mathrm{f}+1$ ). This procedure is repeated until the criteria of $G_{2 f+l}(i, j) \geq 1$ and $f \leq 9$ are fulfilled. If the criteria in the secondary block filtering have been fulfilled, the pixel at $(i, j)$ is replaced by median value, then the sliding window will be shifted to the next pixel, $f$ returns to the initial value and the ouput pixel becomes

$$
Y(i, j)=(1-b) \cdot M_{b}(i, j)
$$

$M_{a}(i, j)$ and $M_{b}(i, j)$ denote the median value for main block filtering and secondary block filtering, respectively, in accordance with eq. (8). 


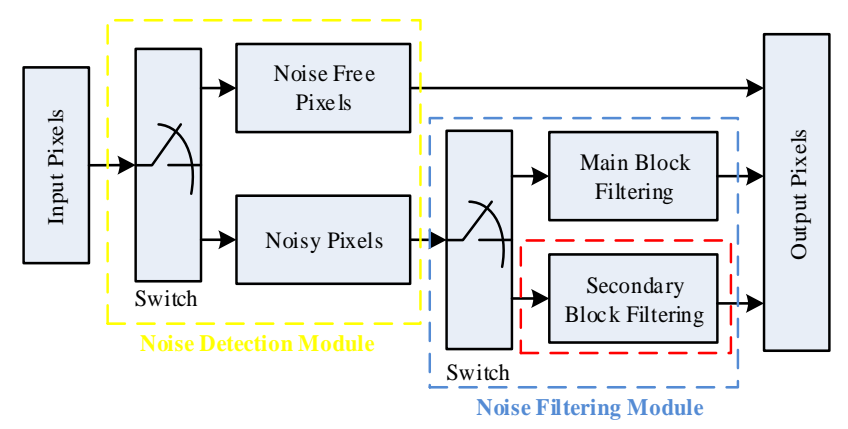

Fig. 3. Architecture of the ASAM algorithm.

\section{RESULTS AND DISCUSSION}

The experiments were performed using standard testing image and real neutron radiographic images. The results are presented and compared to the results of well-established algorithms for impulse noise removal based on switching methods, namely noise adaptive fuzzy switching median (NAFSM) filter [30], decision-based algorithm (DBA) [31], and adaptive switching non-local mean (NASNLM) [19].

The standard testing image results are evaluated based on such noise removal qualities as peak signal-to-noise ratio (PSNR) and structural similarity (SSIM) index [32]. For real application images, the results are measured based on signal-tonoise ratio (SNR) [33]. The PSNR is calculated using eq. (11) as follows:

$$
P S N R=10 \log _{10}\left(\frac{L^{2}}{M S E}\right)
$$

where $L$ denotes the dynamic intensity range of image pixels, and the MSE denotes the mean squared error as expressed in eq. (12).

$$
M S E=\frac{1}{M N} \sum_{i=1}^{M} \sum_{i=1}^{N}\left(y_{i j}-x_{i j}\right)^{2}
$$

Here, $M$ and $N$ are the dimensions of the image, $y_{i j}$ is the pixel intensity at location $(i, j)$ in the original image, and $x_{i j}$ is the pixel intensity at location $(i, j)$ in the filtered image. follows:

The SSIM is calculated using eq. (13) as

$$
\operatorname{SSIM}(x, y)=\left(\frac{2 \mu_{\mathrm{x}} \mu_{\mathrm{y}}+\mathrm{C}_{1}}{\mu_{\mathrm{x}}^{2}+\mu_{\mathrm{y}}^{2}+\mathrm{C}_{1}}\right)^{\alpha}\left(\frac{2 \sigma_{\mathrm{x}} \sigma_{\mathrm{y}}+\mathrm{C}_{2}}{\sigma_{\mathrm{x}}^{2}+\sigma_{\mathrm{y}}^{2}+C_{2}}\right)^{\beta}\left(\frac{\sigma_{\mathrm{xy}}+C_{3}}{\sigma_{\mathrm{x}} \sigma_{\mathrm{y}}+C_{3}}\right)^{\gamma}
$$

where $\mu_{\mathrm{x}}$ and $\mu_{\mathrm{y}}$ denote the average of the original and the denoising image intensities, respectively, $\sigma_{x}$ and $\sigma_{y}$ denote the standard deviation of the original and the denoising image, respectively, $\sigma_{\mathrm{xy}}$ is the cross-correlation between the original image and the denoising image, $\mathrm{C}_{1}=\left(\mathrm{K}_{1} \mathrm{~L}\right)^{2}$ and $\mathrm{K}_{1}=0.01, \mathrm{C}_{2}=\left(\mathrm{K}_{2} \mathrm{~L}\right)^{2}$ and $\mathrm{K}_{2}=0.03, \mathrm{C}_{3}=\mathrm{C}_{2} / 2$, and $\alpha=\beta=\gamma=1$. If two images are similar, the SSIM index is closer to 1 . follows:

The SNR is calculated using eq. (14) as

$$
S N R=10 \log \left(\frac{P_{S}}{P_{N}}\right)
$$

where $P_{s}$ denotes the average power of the signal, which is given by the average value of the pixels in the image, and $P_{N}$ denotes the noise power which is given by the variance of the pixel values in such a region [33].

The experiments were performed in MATLAB R2014a using a PC with Windows 7 operating system, $8 \mathrm{~GB}$ RAM, and an Intel(R) Core(TM) i5-3337U CPU @1.80 GHz.

\section{Simulation results of ASAM}

The 8-bit gray-scale Lena image with a size of $512 \times 512$ pixels is used as a standard test image which contains plenty of textural details, smooth region and border. To simulate the degradation of neutron radiographic images, white spot noise was added to the Lena image by modifying the pepper into salt in salt and pepper noise. The image was corrupted by noise densities varying from $10 \%$ to $95 \%$ and filtered using several denoising methods, namely DBA, NAFSM, NASNLM, and ASAM, respectively. The PSNR results of these filters are showed in Fig. 4.

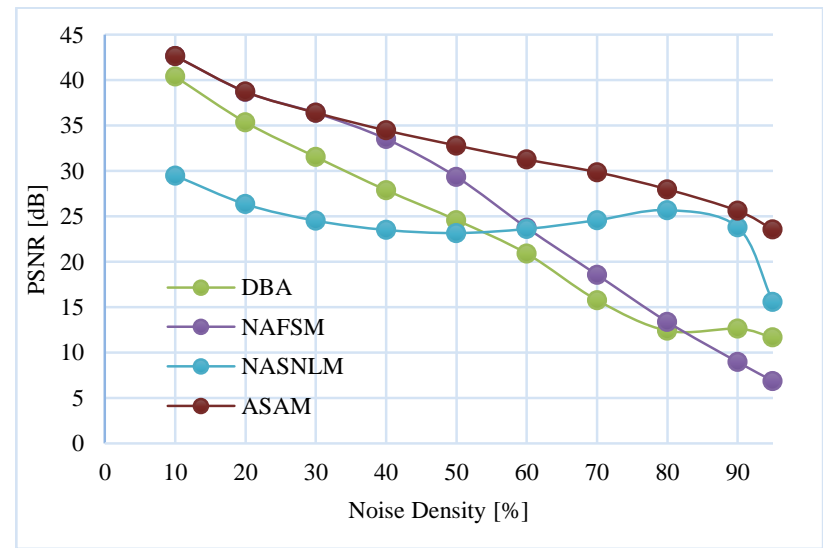

Fig. 4. The PSNR comparison between ASAM and other filters.

It can be seen that the ASAM filter outperformed other filters used in the existing literatures by having the highest PSNR values, especially at noise densities in the $40 \%$ to $95 \%$ range. At noise densities in the $10 \%$ to $30 \%$ range, the PSNR of ASAM filter equal to that of NAFSM, since both filters used a similar method, i.e., simple switching median (SM) filter [29]. However, at higher noise densities, the ASAM filter is switched to secondary block filtering as filtering alternative. ASAM filter removes not only much 
noise but also preserves more details, represented as SSIM results shown in Fig. 5.

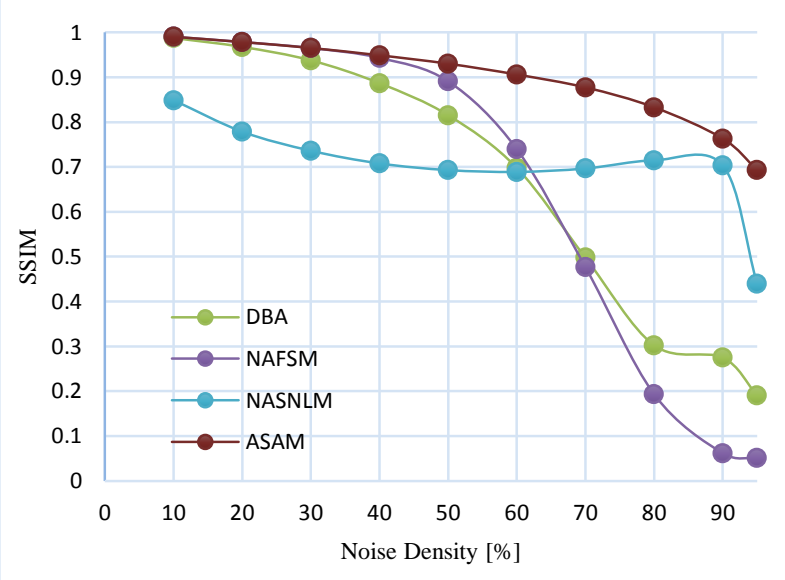

Fig. 5. The SSIM comparison between ASAM and other filters.

The addition of secondary block filtering in ASAM filter resulted in highest similarity level, compared to other filters, especially at noise densities in the $50 \%$ to $95 \%$ range. Clearly, ASAM filter achieves the highest PSNR and SSIM compared to DBA, NAFSM, and NASNLM filters.

Figure 6. shows the restoration results for Lena image that is corrupted with white spot noise of $95 \%$. Visually, it can be seen that the ASAM filter can remove the noise successfully, while other filtering methods could not successfully remove the noise.

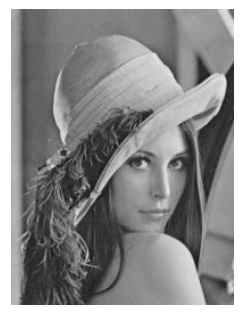

(a)

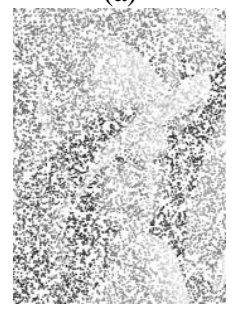

(d)

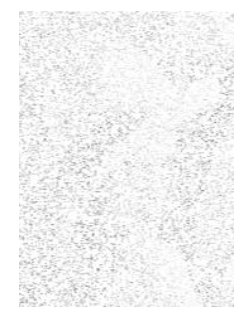

(b)

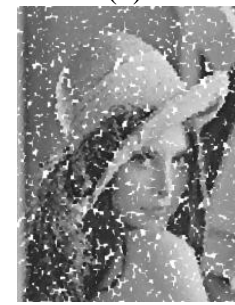

(e)

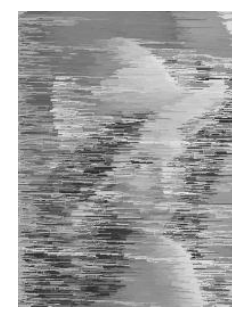

(c)

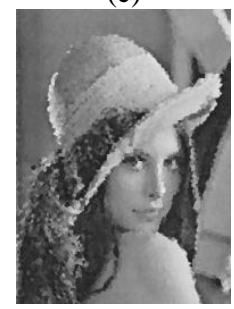

(f)
Fig. 6. (a) Original gray-scale Lena image; (b) Degraded image by WSN of $95 \%$ noise density; (c) Restored image by DBA; (d) Restored image by NAFSM; (e) Restored image by NASNLM; and (f) Restored image by ASAM filter.

We also measured the denoising time for each filtering method in the simulations and presented it graphically in Fig. 7. The ASAM filter performance is better than other algorithms, except for $95 \%$ noise density case. In this case, ASAM filter needs 0.3 seconds longer than NAFSM.

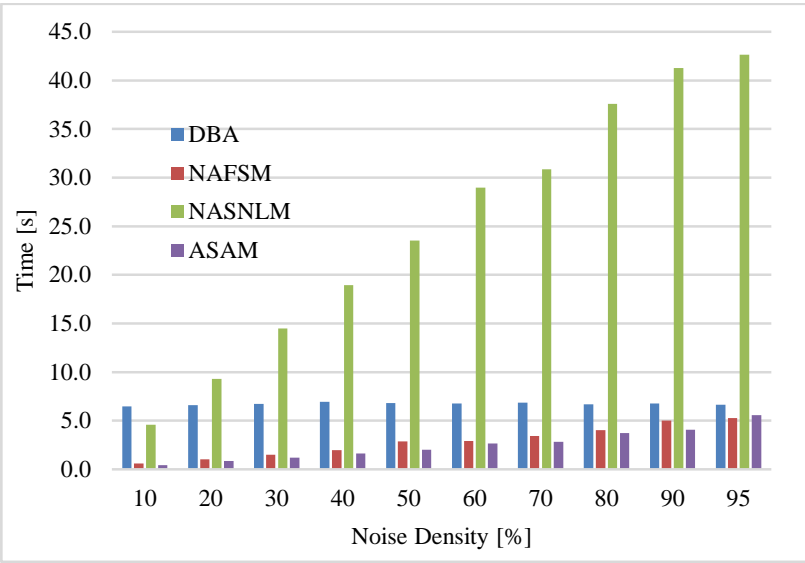

Fig. 7. The denoising time comparison between ASAM and other denoising algorithms.

\section{Real application tests}

In the real application data testing, we used water valve radiographic image as sample data. Noise in the neutron image is changed from random value impulse noise to fixed value impulse noise using threshold module. Then, thresholded result is filtered using DBA, NAFSM, NASNLM, and ASAM filters, respectively.

Figure 8(a) shows the noisy thermal neutron radiographic image of water valve as original image. The image was taken from NRF BATAN using instrumental parameters described in [34] and was acquired in 8 minutes of exposure time. Figure 8(b) shows the noisy image that has been separated from noise and image detail using specified threshold value $T_{0}$ of $30 \%$. The number of noisy pixels that can be detected is 152927 pixels or $14.58 \%$ of the total number of pixels. Figures $8(\mathrm{c})$ to $8(\mathrm{f})$ show the filtering results using each denoising method. Results are compared qualitatively by visual inspection and quantitatively using the signal-tonoise ratio (SNR).

It can be seen that NAFSM and NASNLM filters failed to completely remove white spot noise in the image. Obviously, only the DBA and the proposed ASAM filters are able to produce perceptible restoration images. However, the ASAM attained an SNR that is $1.64 \%$ higher compared to DBA filter. The ASAM filter also resulted in more homogenous image, as presented by its lower standard deviation. 


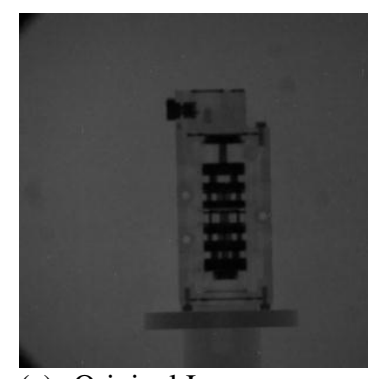

(a) Original Image $\mathrm{SNR}=25.31 \mathrm{~dB}$

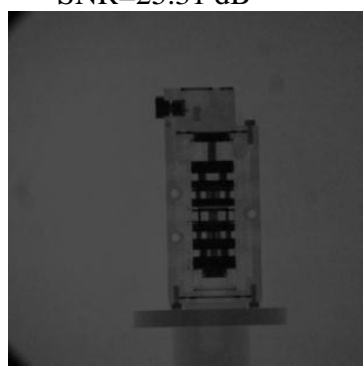

(c) DBA

$\mathrm{SNR}=32.42 \mathrm{~dB}$

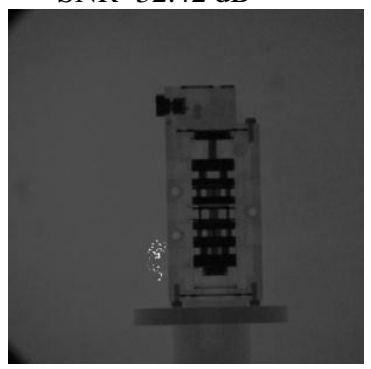

(e) NASNLM

$\mathrm{SNR}=18.77 \mathrm{~dB}$

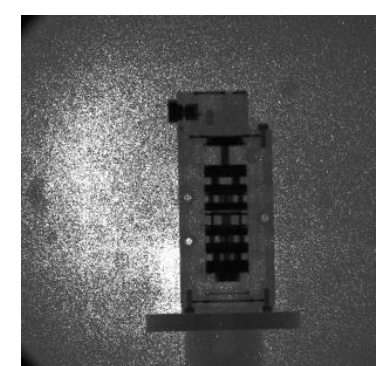

(b) Noisy image threshold $\mathrm{SNR}=5.03 \mathrm{~dB}$

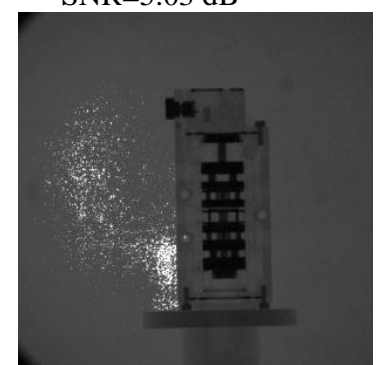

(d) NAFSM

$\mathrm{SNR}=6.01 \mathrm{~dB}$

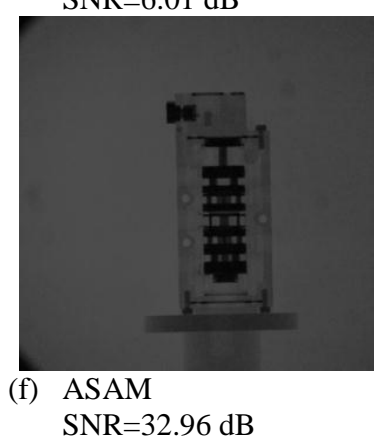

Fig. 8. (a) Valve radiographic image as original image; (b) threshold result; (c) DBA filtering result; (d) NAFSM filtering result; (e) NASNLM filtering result; (f) ASAM filtering result.

The threshold value $T_{0}$ strongly influenced the number of noisy pixels that can be detected. As shown in Table 1, ASAM filter threshold selection affected to the statistics of noise detected in mean, standard deviation, and SNR parameters.

Table 1. Effect of Threshold $T_{0}$ in ASAM filtering.

\begin{tabular}{ccccc}
\hline $\boldsymbol{T}_{\boldsymbol{\theta}}(\%)$ & $\begin{array}{c}\text { Number of } \\
\text { Noise } \\
\text { Detected (\%) }\end{array}$ & Mean & $\begin{array}{c}\text { Standard } \\
\text { Deviation }\end{array}$ & SNR (dB) \\
\hline 50 & 0.017 & 77.880 & 4.381 & 25.00 \\
45 & 0.024 & 77.877 & 4.362 & 25.03 \\
40 & 0.052 & 77.858 & 4.296 & 25.16 \\
35 & 0.685 & 77.591 & 3.714 & 26.40 \\
30 & 14.584 & 75.460 & 1.750 & 32.96 \\
\hline
\end{tabular}

As presented in Table 1, it can be seen that for $\mathrm{T}_{0}=30 \%$, ASAM filtering achieved the highest SNR with highest spatial homogeneity with lowest standard deviation.

\section{CONCLUSION}

In this paper, we proposed ASAM filter for white spot noise removal of neutron radiographic image. The ASAM filter is able to suppress highdensity white spot noise and at the same time preserve fine image details. In the simulation using standard (Lena) and real application (neutron radiographic) images, ASAM filtering results are superior to other filtering method results. Standard image simulation showed that for denoising of white noise at the highest noise level of $95 \%$, the PSNR of DBA, NAFSM, NASNLM, and ASAM were $16.6967 \mathrm{~dB}, 16.696 \mathrm{~dB}, 18.860 \mathrm{~dB}$, and $23.584 \mathrm{~dB}$, respectively. The denoising times for DBA, NAFSM, NASNLM, and ASAM were $6.469 \mathrm{~s}$, $5.186 \mathrm{~s}, 36.735 \mathrm{~s}$, and $5.197 \mathrm{~s}$, respectively. Using real application image, the ASAM has performed better than other methods. The SNR of DBA, NAFSM, NASNLM, and ASAM were $32.42 \mathrm{~dB}$, $6.01 \mathrm{~dB}, 18.77 \mathrm{~dB}$, and $32.96 \mathrm{~dB}$, respectively. In general, ASAM filter improves restoration quality of images, especially that has a high density of white noise, and is able to yield good filtering result with efficient running time.

\section{ACKNOWLEDGMENT}

The authors thank the National Nuclear Energy Agency (BATAN) for the support facility given by the Laboratory of Neutron Radiography and Tomography Installation (FRTN). We also thank for doctoral scholarship support from the Ministry of Research and Technology (KEMENRISTEK) of the Republic of Indonesia.

\section{REFERENCES}

1. A.E. Craft, G.C. Papaioannou, D.L. Chichester et al., Phys. Procedia 88 (2017) 81.

2. Q. Shuang, Z. Wei-jing and S. Jia-ning, Nucl. Instrum. Methods Phys. Res. A (2015) 1.

3. M. Rezazadeh, H. Jafari, S.A.H. Feghhi et al., Prog. Nucl. Energy 87 (2016) 20.

4. A.C. Madden, R.C. Schirato, A.L. Swift et al., IEEE Trans. Nucl. Sci. 64 (2017) 1810.

5. A. Datta and A.I. Hawari, IEEE Trans. Nucl. Sci. 64 (2017) 1652.

6. D.E. Pooley, J.W.L. Lee, M. Brouard et al., IEEE Trans. Nucl. Sci. 64 (2017) 2970.

7. P.C. Hungler, L.G.I. Bennett, W.J. Lewis et al., Phys. Procedia 43 (2013) 169. 
8. F.C. de Beer, F. Gruenauer, J.M. Radebe et al., Phys. Procedia 43 (2013) 34.

9. J. Soltes, L. Viererbl, Z. Lahodova et al., IEEE Trans. Nucl. Sci. 63 (2016) 1640.

10. F. Utaminingrum, K. Uchimura and G. Koutaki, Proc. 1st IEEE/IIAE Int. Conf. Intell. Syst. Image Process. 2013 (2013) 111.

11. H.H. Chou, L.Y. Hsu and H.T. Hu, IEEE Trans. Cybern. 43 (2013) 296.

12. X. Qi, B. Liu and J. Xu, J. Vis. Commun. Image Represent. 36 (2016) 1.

13. P. Karthikeyan and S. Vasuki, J. Circuits. Syst. Comput. 25 (2016) 1650130.

14. X. Wang, S. Shen, G. Shi et al., J. Vis. Commun. Image Represent. 38 (2016) 440.

15. A. Roy, J. Singha, S.S. Devi et al., Signal Processing 128 (2016) 262.

16. M. Habib, A. Hussain, S. Rasheed et al., AEU Int. J. Electron. Commun. 70 (2016) 689.

17. O.S. Faragallah and H.M. Ibrahem, AEU - Int. J. Electron. Commun. 70 (2016) 1034.

18. S.J. Horng, L.Y. Hsu, T. Li et al., J. Vis. Commun. Image Represent. 24 (2013) 956.

19. M. Nasri and S. Saryazdi, Sci. Iran. 20 (2013) 760.

20. V.S. Bhadouria, D. Ghoshal and A.H. Siddiqi, Signal, Image Video Process. 8 (2014) 71.

21. T. Veerakumar, S. Esakkirajan and I. Vennila, Signal, Image Video Process. 8 (2014) 159.
22. J. Varghese, M.S. Khan, M. Siddappa et al., IET Image Process. 8 (2014) 199.

23. X. Zhang, F. Ding, Z. Tang et al., AEU - Int. J. Electron. Commun. 69 (2015) 307.

24. S. Deivalakshmi and P. Palanisamy, AEU - Int. J. Electron. Commun. 70 (2016) 757.

25. P. Aggarwal, H. Kaur and N. Goel, Res. Cell An Int. J. Eng. Sci. 17 (2016) 2229.

26. A. Hindasyah, D. Sudiana and D. Gunawan, Int. J. Adv. Comput. Sci. Technol. 4 (2015) 1.

27. H. Li, B. Schillinger, E. Calzada et al., Nucl. Instrum. Methods Phys. Res., Sect. A 564 (2006) 405.

28. K. Osterloh, T. Bucherl, C.L. von Gostomski et al., Nucl. Instrum. Methods Phys. Res., Sect. A 651 (2011) 171.

29. H. Ibrahim, N. Sia, P. Kong et al., IEEE Trans. Consumer Electron. 54 (2008) 1920.

30. K. Kal, V. Toh, S. Member et al., IEEE Signal Process. Lett. 17 (2010) 281.

31. K.S. Srinivasan and D. Ebenezer, IEEE Signal Process. Lett. 14 (2007) 189.

32. A.C. Bovik, The Essential Guide to Image Processing, Academic Press, California (2009) 571.

33. G. Dougherty, Digital Image Processing for Medical Applications, Cambridge University Press, New York (2009) 37.

34. Bharoto, Atom Indonesia 39 (2013) 95. 